\title{
Management of Atrial Fibrillation in Recipients of Cardiac Resynchronization Therapy
}

\author{
Jan Ciszewski ${ }^{1}$ and Maciej Sterliński²
}

1. 2nd Department of Cardiac Arrhythmia, National Institute of Cardiology, Warsaw, Poland; 2. 1st Department of Cardiac Arrhythmia, National Institute of Cardiology, Warsaw, Poland

DOI: https://doi.org/10.17925/EJAE.2021.7.1.19

\begin{abstract}
A S atrial fibrillation (AF) and heart failure (HF) often co-exist and influence each other, the problem of appropriate AF management concerns a wide group of recipients of cardiac resynchronization therapy (CRT). Mutual correlations between AF treatment and CRT efficacy are even closer and more complex than the pure AF and HF coexistence. The AF paroxysm, with an uncontrolled and high ventricular rate, hinders the ability of CRT to deliver a sufficient percentage of effectively resynchronized ventricular paced beats, resulting in $\mathrm{HF}$ exacerbation, which, in turn, may promote further AF progression. In this context, the proper management of AF in patients undergoing CRT is of supreme importance. However, there is a shortage of evidence assessing different AF treatment strategies specific to this intriguing subgroup of patients. This article summarizes the current state of knowledge about the AF-CRT relationship and the efficacy of different AF treatment strategies in the context of CRT in order to provide some hints for a proper treatment.
\end{abstract}

\section{Keywords}

Cardiac resynchronization therapy, atrial fibrillation, heart failure, atrioventricular node ablation, pulmonary vein isolation

Disclosure: Jan Ciszewski has no financial or non-financial relationships or activities to declare in relation to this article. Maciej Sterliński has received proctoring, consulting, lecturer or investigator fees from Abbot, Biotronik, Boston Scientific, HammerMed and Medtronic

Review Process: Double-blind peer review.

Compliance with Ethics: This article involves a review of the literature and did not involve any studies with human or animal subjects performed by any of the authors.

Authorship: The named authors meet the International Committee of Medical Journal Editors (ICMJE) criteria

for authorship of this manuscript, take responsibility for the integrity of the work as a whole, and have given final approval for the version to be published.

Access: This article is freely accessible at touchCARDIO.com (c) Touch Medical Media 2021.

Received: 29 October 2020

Accepted: 7 January 2021

Published Online: 27 July 2021

Citation: European Journal of Arrhythmia \& Electrophysiology. 2021;7(1):19-25

Corresponding Author: Jan Ciszewski, National Institute of Cardiology, 42 Alpejska Street, 04-628 Warszawa, Poland. E: jciszewski@ikard.pl

Support: No funding was received in the publication of this article.
Atrial fibrillation (AF) and heart failure (HF) often co-exist. The incidence of AF is estimated to reach $45 \%$ of patients with HF and rises with the severity of HF symptoms, from about $5 \%$ of patients in the New York Heart Association (NYHA) class I to 50\% in NYHA class IV. ${ }^{1-4}$ Consistently, the incidence of $A F$ in patients treated with cardiac resynchronization therapy (CRT), which is one of the most powerful therapies of HF in a selected subgroup of patients, is also high, reaching from $25 \%$ to more than $50 \%$ of CRT recipients. ${ }^{5-8}$ However widespread it is, the management of patients with CRT and concomitant AF has many peculiarities, which are different to the treatment of either patients with CRT and sinus rhythm (SR) or patients with AF but no CRT. The aim of this review is to address these peculiarities in the context of the evidence available, and to provide some tips for proper treatment.

\section{Atrial fibrillation: A marker of poorer prognosis and poorer response to cardiac resynchronization}

CRT is unequivocally one of the most potent HF therapies leading to the reduction of symptoms, the number of HF hospitalizations and the total mortality in a selected subgroup of patients with $\mathrm{HF}^{4,9}$ Widely accepted criteria for a CRT device with defibrillation function (CRT-D) implantation include left ventricular ejection fraction (LVEF) $\leq 35 \%$ and QRS complex prolongation varying from $130 \mathrm{~ms}$ in the presence of the left bundle branch block (LBBB) to $150 \mathrm{~ms}$ in the setting of non-LBBB interventricular conduction abnormalities. ${ }^{4,9,10} \mathrm{CRT}$-only pacing (CRT-P) is recommended in patients with mildly reduced LVEF (35-50\%) and indications for cardiac pacing, especially when a significant amount of ventricular pacing is anticipated. ${ }^{11}$ However, the usefulness of CRT in the subset of patients with concomitant AF remains not so evident. Indeed, the randomized controlled trials that led to a wide use of CRT - that is, the MADIT-CRT (ClinicalTrials.gov identifier: NCT00180271) and the REVERSE (ClinicalTrials.gov identifier: NCT00271154) studies and others - were practically restricted to patients with SR. ${ }^{12-17}$ Studies evaluating the role of CRT in patients with concomitant AF bring inconsistent results. However, they unanimously indicate that the presence of $A F$ in a patient undergoing CRT is a risk factor for a poorer response to CRT and a poorer clinical course of HF.

One of the first prospective, randomized trials dedicated to CRT treatment in patients with HF and AF was the MUSTIC study. It showed that patients with HF and functional NYHA class III and LVEF $<35 \%$ profit from biventricular pacing (BiVp) in terms of a longer 6-minute walk test (6MWT) distance, a higher peak oxygen uptake $\left(\mathrm{VO}_{2}\right.$ max) and $70 \%$ fewer hospitalizations compared with controls. ${ }^{18}$ However, the study had major limitations. It recruited patients with AF and a low ventricular rate necessitating a pacemaker implantation, and the paced right ventricular QRS 
complex duration had to be at least $180 \mathrm{~ms}$. As a result, the control group consisted of patients receiving a high percentage of unphysiological right ventricular pacing (RVp), which has a detrimental effect on the LVEF and on the incidence of $A F$, and is associated with a higher risk of HF hospitalizations, especially in patients with reduced LVEF.19-25 Moreover, it was a crossover study, and both of its phases were completed in only $58 \%$ of patients, as the Bivp was preferred by the study participants.

The RAFT trial was the most well-known CRT trial that included patients with $\mathrm{AF}^{26}$ It included patients with $\mathrm{HF}$ and functional NYHA class II or III, an LVEF $<30 \%$, a QRS duration longer than $120 \mathrm{~ms}$ and a mean ventricular rhythm of 60-90 beats per minute who were randomly assigned to receive an implantable cardioverter-defibrillator (ICD) alone or a CRT + ICD (CRT-D) device. The sub-analysis of this study, involving more than 200 patients with permanent AF, revealed no benefit of CRT-D over ICD with regard to the primary composite endpoint of death or HF hospitalizations (hazard ratio [HR] 0.96; 95\% confidence interval [Cl], 0.65-1.41; $p=0.82$ ), cardiovascular death (HR 0.97; 95\% Cl, 0.55-1.71; $\mathrm{P}=0.91$ ) and $6 \mathrm{MWT}$ distance (CRT-D $19 \pm 84 \mathrm{~m}$ versus ICD $16 \pm 76 \mathrm{~m}$; $\mathrm{p}=0.88$ ) during a mean follow-up period of 40 months. Only a trend for fewer HF hospitalizations ( $\mathrm{HR} 0.58$; 95\% $\mathrm{Cl}, 0.38-1.01 ; \mathrm{p}=0.052$ ) and greater improvement in quality of life in the CRT-D group were observed. ${ }^{27}$

These disappointing results are supported by the COMPANION trial, which involved 1,520 patients with HF in NYHA class III or IV, LVEF <35\% and QRS $>120 \mathrm{~ms}$ that were in SR at the baseline visit. The study compared both CRT-D and CRT-P to optimal medical therapy only. In the general population, the study showed better outcomes associated with the presence of both CRT-D and CRT-P devices, compared with optimal medical therapy. ${ }^{28}$ However, Kalscheur et al. evaluated COMPANION study participants based on the presence or absence of a history of paroxysmal AF or atrial flutter (AFI). ${ }^{29}$ In contrast to patients without a history of arrhythmias, in the subgroup with a history of paroxysmal AF/AFI $(n=293)$, the presence of CRT-P/CRT-D did not result in better survival or a lower risk of the composite endpoint of death and hospitalizations. Moreover, the presence of AF/AFI paroxysm during follow up was associated with a greater risk of death or all-cause hospitalizations ( $\mathrm{HR} \mathrm{1.54;95 \%} \mathrm{Cl,}$ 1.26-1.89; $\mathrm{p}<0.001)$ and a greater risk of death or HF hospitalizations (HR 1.86; 95\% Cl, 1.46-2.38; $p<0.001) .{ }^{29}$

These were followed by several observational studies and meta-analyses. Regarding an acute response, most of the trials demonstrated the beneficial role of CRT. They were summarized in a meta-analysis by Upadhyay et al., who showed that the CRT implantation increases 6MWT distance, elevates LVEF and results in a better quality of life, as assessed by the Minnesota Living with Heart Failure Questionnaire in patients with $A F^{30}$ However, the extent of response to CRT remains greater in $S R$ than patients with $A F$, and $A F$ is related to an increased risk of non-responders. ${ }^{30,31}$

Other observational studies have evaluated the influence of CRT on clinical outcomes. Although they have brought inconsistent conclusions, the majority of them were in line with the RAFT and COMPANION trial results, showing either no benefit of CRT over ICD devices in terms of mortality and hospitalization risk or increased mortality in patients with AF versus SR receiving CRT. ${ }^{32-35}$ While there is some evidence showing no discrepancies between patients with AF and SR receiving CRT, the meta-analyses summarizing the efficacy of CRT in patients with HF and AF are in line with the major RCTs and registries showing higher mortality in patients with AF receiving CRT and no mortality reduction after implantation of the CRT device. ${ }^{31,36-38}$

\section{The influence of atrial fibrillation on the efficacy of cardiac resynchronization therapy and biventricular pacing percentage: The more, the better}

Many hypothetical mechanisms exist resulting in a lower response to CRT, a higher risk of HF progression and higher mortality in patients with AF. Even though AF may be regarded as a marker of HF severity, the occurrence of AF during CRT leads to an inability to establish the optimal atrio-ventricular (AV) delay, a higher ventricular rate, and variable AV conduction, providing a higher percentage of conducted ventricular sensed beats limiting the percentage of correctly resynchronized Bivp beats. Moreover, it bears a higher risk of appropriate and inappropriate shocks and a worse clinical outcome, especially during AF associated with a high ventricular rate. ${ }^{33,39}$

There is a growing body of evidence that the percentage of Bivp (Bivp\%) is inversely correlated with mortality and the risk of $\mathrm{HF}$ progression. ${ }^{5,6400-42}$ According to the latest studies, the minimal BiVp\% in patients with AF, assuring a similar prognosis to patients without $A F$, is $98 \% .{ }^{5.6} \mathrm{As}$ a result, the optimal Bivp\% that is currently recommended should be as close to $100 \%$ as possible. ${ }^{4}$ Moreover, some authors argue that every day spent with a Bivp\% < 95 can alter patients' prognoses, even though it may not significantly influence the overall BiVp\%. Based on a prospective registry of patients undergoing CRT that were monitored remotely, Mazurek et al. have shown that the cumulative burden of days with low Bivp\% (i.e. the number of days with Bivp\% <95) is associated with the risk of death. ${ }^{41}$ The correlation was irrespective not only of the typical confounding factors such as age, gender, renal function, ischaemic HF and the presence of LBBB, NYHA class and LVEF, but also of the AF burden and the cumulative Bivp\%.

Inadequate Bivp\% is a common finding in one- to two-thirds of patients undergoing CRT. ${ }^{6,39}$ Additionally, the Bivp\% value presented by a CRT device itself may be overestimated. In the older study by Kamath et al., Holter monitoring revealed that in a population of patients with a mean Bivp\% of 94.5 reported by the device, up to $50 \%$ of patients had an inadequate Bivp\% of $<90$ due to widespread occurrence of fused and pseudo-fused beats. ${ }^{43}$ These results were recently confirmed in a study by Hernández-Madrid et al. ${ }^{44}$ However high the intersubject variability was, the mean device overestimation of the effective Bivp\% in the study reached $7 \%{ }^{44}$ Moreover, the overestimation of the Bivp\% may be caused by the use of built-in device algorithms made to enhance left ventricular (LV) or BiVp\%, such as conducted AF response, ventricular rate regulation, EffectivCRTTM (Medtronic, Dublin, Ireland) during $A F$ algorithms or LV-triggered function. ${ }^{45-48}$ The role of these algorithms has not been well established, and the presence of fused beats cannot be considered as fully effective resynchronization.

The potent influence of BiVp\% may be an explanation for the disappointing results of the studies showing no benefit of CRT in patients with AF. Indeed, in the RAFT trial, a Bivp\% of >95 was only observed in $34.3 \%$ of patients with AF treated with $\mathrm{CRT}^{27}$ Moreover, only one subject in the AF CRT-D subgroup underwent atrioventricular node ablation (AVNA) at the investigators' discretion. Similar results have been reported in other studies. ${ }^{6,35,36}$ There is no information in other studies about BiVp\% at all. ${ }^{29,32,33}$

Based on this evidence, in the setting of patients with AF and HF with classical indications for CRT (i.e. HF with reduced LVEF, LVEF $<35 \%$ and QRS prolongation $>130 \mathrm{~ms}$ ), current guidelines recommend the implantation of a CRT device only if high Bivp\% is anticipated or a strategy to ensure high Bivp\% is implemented. 


\section{The role of atrioventricular node ablation in patients with atrial fibrillation undergoing cardiac resynchronization therapy}

Apart from uptitrating the dose of negative chronotropic and dromotropic drugs, which has a limited effect, another way to ensure high BiVp\% beats during AF is by performing an AVNA. This relatively simple procedure is highly effective by making the ventricular rhythm completely independent of the atrial tachyarrhythmias and by ensuring almost 100\% Bivp (in patients without a high burden of ventricular rhythm disturbances, which also may affect the BiVp\%). Indeed, there is strong evidence that performing AVNA enhances the CRT performance in patients with $\mathrm{HF}$ and $\mathrm{AF}$, and makes their clinical course similar to those in SR.

In their two prospective, observational, early studies in 2006 and 2008, Gasparini et al. reported that the extent of the response to CRT and the survival rate in patients with $\mathrm{AF}$ is strongly dependent on whether or not the AVNA procedure was performed. 49,50 The patients who had undergone CRT with AF in addition to AVNA performed similarly to the patients with CRT and SR, and did significantly better than the patients with AF who were treated with drugs that ensured Bivp\% of $>85 .{ }^{49,50}$ These findings were then supported in the CERTIFY trial; a multicentre, prospective, observational study of 7,384 consecutive patients who had undergone CRT from centres in Italy, France, switzerland, Germany and the UK. ${ }^{51}$ The study compared clinical outcomes of patients with permanent AF in whom AVNA was performed (AVNA+, $n=443$ ) or not (AVNA-, $n=895$ ) to those in SR $(n=6,046)$. During a median of 37 months of follow-up the overall mortality and cardiac mortality was similar in the AVNA+ and the SR groups (6.8 versus 6.1 per 100 person-years, and 4.2 versus 4.0, respectively, $p=$ not significant for both), even though the AVNA+ group tended to be older and were in a higher NYHA class. Moreover, mortality and cardiac mortality in the AVNA- group was significantly higher compared to either the SR or the AVNA+ groups, reaching 11.3 and 8.1 per 100 person-years, respectively. Based on these results, the authors concluded that AVNA was associated with a reduction in mortality and cardiovascular mortality of $>50 \%$. These relations persisted in the multivariable analysis that included confounding factors such as age, sex, coronary artery disease, NYHA functional class, the presence of a CRT-D (or CRT-P) device, LVEF and QRS duration. Of note, there were significant differences between the groups in BiVp\% assessed 12 months after recruitment, with the highest Bivp\% beats in the AVNA+ group (96\%), followed by the SR group (92\%) and the lowest in the AVNA-group (87\%) $(p<0.001)$. However, Bivp\% was assessed in a limited number of the CERTIFY participants and should be interpreted with caution. ${ }^{51}$

The results of the trials performed by Gasparini et al. are supported by two retrospective analyses of relatively small studies. ${ }^{52,53}$ Based on a group of 131 patients with CRT, Ferreira et al. showed that in the group of patients with AF but without AVNA there was an increased percentage of CRT non-responders (48\% versus 15\% [AVNA+] versus $21 \%$ [SR], $p<0.008) .{ }^{52}$ Apart from age, the lack of AVNA was the only independent risk factor for death and HF hospitalization. The results in the AVNA+ group were similar to those in patients in SR. Importantly, the group of patients with AF included not only those with a permanent form of AF but also patients with a history of a paroxysmal and persistent form of arrhythmia. Dong et al. divided 154 consecutive patients with $A F$, referred for the CRT-D implantation, into those who underwent AVNA and those who received medical therapy only. ${ }^{53}$ Consistent with the aforementioned trials, they found that the patients who underwent AVNA responded better to CRT (in terms of the amelioration of LVEF, LV end-systolic diameter and the NYHA functional class) and had a markedly lower risk of death ( $\mathrm{HR} 0.13 ; 95 \% \mathrm{Cl}, 0.03-0.58, \mathrm{p}=0.007$ ) and the risk of combined death, heart transplant and an LV assist device (HR 0.19; 95\% Cl, 0.06-0.62; $p=0.006$ ).

In contrast to these studies, a recent study by Mittal et al. of the Medicare and MarketScan registries including more than 24,000 patients found that in patients with AF treated with CRT ( $n=588)$, AVNA was associated with fewer AF hospitalizations, but the HF hospitalization risk remained the same in the AVNA+ and AVNA- groups. ${ }^{54}$ While conflicting evidence suggesting a poorer outcome in the patients undergoing CRT and AVNA exists, a recent meta-analysis supports the notion that performing AVNA in patients with AF receiving CRT markedly increases their response to CRT and decreases the risk of death and hospitalizations, making the survival benefit comparable to that in patients with SR and CRT. ${ }^{54-56}$

The major disadvantage of the evidence assessing the role of AVNA in patients undergoing CRT with AF is the observational nature of the studies, which makes them prone to selection bias. In many of them there is a lack of reliable data regarding Bivp\%, making it impossible to assess to what extent the beneficial effect of AVNA is related to the rise in BiVp\%. ${ }^{35,52,54}$ Moreover, AVNA should be regarded as a palliative procedure, which renders patients CRT/pacemaker-dependent. Indeed, current guidelines do not recommend AVNA as a routine practice in every patient with $\mathrm{HF}$ and $\mathrm{AF}$ (even permanent AF) undergoing CRT implantation.4,9,10 Certainly, new evidence based on prospective, randomized trials in this field is urgently needed. However, based on the current knowledge, it seems reasonable to recommend that in patients treated with CRT with low BiVp\% (i.e. $<98 \%$ ), in whom the loss of the BiVp is attributed to AF, AVNA should be considered.

\section{Cardiac resynchronization in patients with atrial fibrillation with indications for pacing}

The evidence assessing CRT efficacy in patients with an indication for pacing in the subgroup of patients with AF is certainly stronger and more concordant with the studies concerning patients in SR. Apart from the aforementioned MUSTIC study, another trial that compared BiVp to RVp-only was the HOBIPACE study. ${ }^{18,57}$ It involved patients with a marked LV dysfunction and indications for ventricular pacing but with no interventricular conduction abnormalities at baseline. Although relatively small, it showed a clear benefit of CRT over RVp-only in patients in SR and in those with AF associated with the reduction of LV volume, the levels of NT-proBNP (N-terminal pro B-type natriuretic peptide), the increase in LVEF and exercise tolerance, and the amelioration of quality of life.

A similar effect of CRT compared with RVp was also shown in the subgroup of patients with HF and mildly reduced LVEF. The BLOCK-HF trial (ClinicalTrials.gov identifier: NCT00267098) recruited patients with class I or class Ila indications for pacing due to high-degree atrioventricular block, and LVEF $<50 \%$ associated with HF symptoms. ${ }^{58,59}$ It showed that BiVp was associated with an improvement in NYHA functional class, an increase in LVEF, a reduction in LV volumes, a greater extent of LV reverse remodelling, an increase in quality of life and a lower risk of an urgent-care visit for HF and composite of death. ${ }^{58-61}$ The effect of CRT was irrespective of the presence of AF. Interestingly, AF was diagnosed in more than half of the study participants, although the presence of persistent atrial tachyarrhythmias was one of the exclusion criteria.58,59

Other studies comparing BiVp with RVp in patients with HF with permanent AF who had undergone AVNA also showed the superiority of CRT over RVp in terms of better exercise tolerance, an improvement in LV echocardiographic parameters and a reduction in hospitalizations 
and mortality risk. ${ }^{18,62,63}$ Based on these studies and the evidence showing that the risk of a deleterious effect of RVp correlates with higher ventricular pacing percentage and with the reduction of LVEF, it is widely accepted that in patients with AF and indications for pacing, CRT rather than RVp should be preferred irrespective of the native QRS duration, provided the LVEF is $<50 \%$ and a high percentage of ventricular pacing $(>40 \%)$ is anticipated. ${ }^{11,25,59,6,6,65}$

\section{Rate control or rhythm control strategy in patients with atrial fibrillation and cardiac resynchronization therapy}

Regardless of the long-standing and vigorous debate about the superiority of either the rhythm control or the rate control strategy in AF treatment, there is still no convincing evidence that one is better than the other in terms of a better prognosis in the general population of patients with $\mathrm{AF}^{66-68}$ Indeed, the choice of the rhythm control strategy (catheter ablation, preferably) is mandated mainly for quality-of-life improvement. That is why the indispensable condition for pulmonary vein isolation $(\mathrm{PVI})$ in the general population of patients with $\mathrm{AF}$ is the presence of symptomatic $A F^{68}$ However, there is a growing body of evidence that in patients with HF, the rhythm control strategy may positively influence their prognosis. As a result, PVI in patients with HF obtained a class Ila recommendation (and class I in specific cases) in the recent European Society of Cardiology (ESC) guidelines for AF management. ${ }^{68}$

\section{The role of cardiac resynchronization therapy in the} development of atrial fibrillation

choosing between rate and rhythm control strategies would be made easier if it was known whether CRT itself has any effect on the natural course of AF. Hypothetically, CRT-driven reverse remodelling - which is not only limited to an LVEF rise but also may lead to the reduction of mitral regurgitation (which is caused by LV dilatation), left atrial diameter and atrial strain - could limit AF development. This mechanism could apply at least to cases where the occurrence of $A F$ is regarded as a marker of HF progression. ${ }^{69,70}$ Indeed, some studies support this hypothesis. In a follow-up of CRT recipients with AF regarded as permanent who underwent AVNA, Gasparini et al. found that approximately $10 \%$ of the study population experienced spontaneous SR resumption, mainly within 4.4 months after the CRT implantation (in some rare cases, AF could stop even after 3 years of CRT). ${ }^{71}$ These observations are supported by Delnoy et al., who reported spontaneous SR resumption in $25 \%$ of patients undergoing CRT.72

Contrary to a possible protective effect of CRT implantation on persistent $A F$, several studies, including sub-analyses of the CARE-HF (ClinicalTrials.gov identifier: NCT00170300) and the RAFT (ClinicalTrials.govidentifier:NCT00251251) trials, have shown no influence of CRT implantation on the de novo occurrence of AF in patients with $\mathrm{HF}^{27,31,73-77}$ On the other hand, it seems that high-quality resynchronization (by the implementation of algorithms ensuring continuous optimization of resynchronization) has a positive effect on AF occurrence. ${ }^{78,79}$ In recent studies, it has been shown that the AdaptivCRT algorithm lowers the incidence of clinically significant AF paroxysms compared with conventional, echocardiographically optimized CRT. ${ }^{78,79}$ The AdaptivCRT is an algorithm that continuously optimizes atrioventricular and ventriculo-ventricular intervals, and promotes LV-paced fusion beats with the intrinsically conducted ventricular depolarization when atrio-ventricular conduction is normal. In effect, the algorithm lowers the amount of RVp beats, which (as mentioned before) was shown to promote $A F^{78,79}$ However, it is worth noting that the implementation of the AdaptivCRT algorithm is restricted to patients with prevalent SR (as it is based on the assessment of AV interval); it is useless in patients with persistent or permanent AF.

\section{Rate control strategy \\ Taking into account:}

- the crucial role of adequate BiVp\% to ensure CRT efficacy in patients with $A F$;

- the aforementioned studies suggesting a similar prognosis of AF plus AVNA to SR subgroups among CRT recipients;

- the fact that $\mathrm{AF}$ in patients with $\mathrm{HF}$ is often regarded as a marker of $\mathrm{HF}$ severity rather than an independent entity as well as evidence; and

- that HF is a risk factor for rhythm control failure;

the choice for a rate control strategy while dealing with AF episodes in a CRT recipient seems a reasonable option..$^{80}$ According to general guidelines on HF treatment, the first-line option should be the intensification of pharmacotherapy (mainly beta blockers and digoxin, whereas calcium channel blockers are contraindicated in patients with reduced LVEF). ${ }^{9}$ The decision to perform AVNA should be made only after the failure/incomplete efficacy of a drug treatment.9.68 However, considering the results of the studies assessing the efficacy of CRT in patients undergoing AVNA+ versus AVNA-, the decision to perform the ablation procedure should not be postponed.

Moreover, the combination of AVNA and CRT also may be useful as a rate control treatment of AF in patients with narrow QRS at baseline. In the APAF-CRT trial (ClinicalTrials.gov identifier: NCT02137187), Brignole et al. compared the pace and ablate strategy to optimal medical therapy in patients with AF and narrow QRS who were not eligible for catheter ablation of $\mathrm{AF}^{4,81}$ The authors found that the CRT plus AVNA group had fewer HF hospitalizations and lower mortality than the patients treated with optimal medical therapy. Although observed in the whole study cohort, these differences were noticed mostly in the LVEF $<35 \%$ subgroup, whereas symptom relief was marked especially in the LVEF $>35 \%$ subgroup.

In summary, in patients with AF who have been treated with CRT resulting in BiVp\% loss, intensification of the rate control strategy should be initiated. If pharmacotherapy fails and cumulative Bivp\% is still <95-98\%, AVNA should be considered. This should especially apply to patients with persistent AF (especially long-standing persistent AF). AVNA and CRT implantation may also be considered as the last option of the rate control strategy of AF, not only in patients with interventricular disturbances but also in those with narrow QRS.

\section{Rhythm control strategy}

The idea of rhythm control in the subpopulation of patients with HF treated with CRT is especially appealing, while there exists a strict correlation between the presence of $\mathrm{AF}$ and the inability to provide proper HF treatment (associated with the loss of adequate Bivp\%) in this subgroup of patients. Spontaneous SR resumption in a patient having undergone CRT - by ensuring proper BiVp\% - brings back successful CRT, which in turn (by the mechanisms of reverse remodelling), may inhibit further AF development. Unfortunately, there are no data specific to this subgroup of patients; the efficacy of the rhythm control strategy in patients with AF treated with CRT may be driven from the trials only assessing rhythm control in the general population of patients with HF. In contrast to the general AF population, there is some evidence that a rhythm control strategy in patients with HF and AF may improve their prognoses. ${ }^{82-84}$

Among antiarrhythmic therapies, the only modality with confirmed efficacy in patients with HF is catheter ablation of AF. Indeed, the trials 
assessing pharmacological rhythm control, including the most recent sub-analysis of the CASTLE-AF trial, have not shown any superiority over rate control treatment in patients with $\mathrm{HF}^{85-87}$ Contrary to antiarrhythmic agents, catheter ablation - with complete pulmonary veins isolation as its essential component - has been proven to reduce AF recurrences and burden, and to ameliorate EF, quality of life and exercise tolerance in patients with HF compared with either rate-slowing drugs or AVNA plus CRT implantation in short-term and mid-term follow-up. ${ }^{88-90}$ Moreover, the supreme effect of catheter ablation in patients with HF is also confirmed in the studies directly comparing catheter ablation with antiarrhythmic drugs. In the AATAC trial, catheter ablation turned out to be better than amiodarone in the treatment of persistent $A F$ in patients with $\mathrm{EF}<40 \%$ in terms of freedom from $\mathrm{AF}$, and lower rates of mortality and unplanned hospitalizations. ${ }^{91}$

The beneficial role of catheter ablation in treating AF in patients with HF seems to be confirmed by the CASTLE-AF trial, which compared the efficacy of catheter ablation with optimal medical therapy of paroxysmal or permanent AF in patients with symptomatic HF, significantly decreased EF (LVEF <35\%) and with ICD/CRT-D.82 After a median follow-up of almost 38 months, the composite endpoint of mortality and HF hospitalizations occurred in significantly fewer patients in the catheter ablation group than the optimal medical therapy group (28.5\% versus $44.6 \%$, respectively; HR 0.62; $95 \% \mathrm{Cl}, 0.43-0.87$; $\mathrm{p}=0.007$ ), and lower cardiovascular mortality was reported in the catheter ablation group (13.4\% versus $25.0 \%$; HR 0.53; 95\% Cl, $0.32-$ $0.86 ; \mathrm{p}=0.01)$. Of note, the medical therapy utilized encompassed both the rhythm- and the rate-controlling agents; however, in the majority of patients (70\%) a rate control strategy was applied. In the subgroup analysis, the results among patients with CRT-D were similar, but it did not reach statistical significance (HR 0.54; 95\% Cl, 0.28-1.04 for the primary endpoint). However, this could be attributed to a low sample size, as patients having undergone CRT-D constituted less than onethird of patients (100/363).

The results of the CASTLE-AF trial were confirmed by the recent sub-analysis of 886 patients of the CABANA trial (ClinicalTrials.gov identifier: NCT00911508), who had a history of HF or reported NYHA class $\geq 11 .{ }^{92}$ Although the CABANA trial turned out to be negative in a general population of patients with $A F$, in this selected subgroup catheter ablation was shown to reduce the primary endpoint (a composite of death, disabling stroke, severe bleeding or cardiac arrest), as well as all-cause mortality with the reduction of AF recurrence rate. ${ }^{83}$

The promising results of the CASTLE-AF trial have been recently questioned by the publication of the AMICA trial (ClinicalTrials.gov identifier: NCT00652522). ${ }^{84,93}$ AMICA was a randomized clinical trial that compared the efficacy of catheter ablation with optimal medical therapy in patients with persistent or long-standing persistentAF (lasting 7 days to 4 years) and markedly reduced LVEF ( $\leq 35 \%)$. The primary endpoint was the LVEF increase from baseline to 1 year. Having recruited 140 patients, the study was terminated prematurely for futility. After 1 year, LVEF increased similarly in both the catheter ablation and the optimal medical therapy groups ( $8.8 \%$ versus $7.3 \%$, respectively; $p=0.36$ ). Similarly, the groups did not differ in laboratory (BNP, NT-proBNP levels), exercise tolerance, quality of life and clinical outcomes. Of note, there was significant reduction in AF burden in catheter ablation compared with the optimal medical therapy arm: 1 year AF burden of 0-5\% was observed in $72 \%$ versus $44 \%$, respectively, although the device-derived data on AF burden were published only in half of the recruited patients (39 of 68 and 36 of 72 , respectively).
The conflicting results of the CASTLE-AF and the AMICA trials are a matter of ongoing debate. The most plausible explanation, as stated by the authors of the AMICA trial, is the difference between patients' baseline characteristics of the two studies. The AMICA study participants were sicker in terms of more advanced NYHA class (NYHA class III or IV: $60 \%$ in the AMICA trial versus $31 \%$ in the CASTLE-AF trial), mean EF (27.6\% versus $32.5 \%$ ), the presence of persistent AF (100\% versus $70 \%$ ) and the prevalence of treatment with CRT-D (43\% versus $27 \%$ ). The authors conclude that greater AF and HF advancement in the AMICA study participants could have prevented catheter ablation efficacy. The subgroup analysis of the CASTLE-AF trial indicates the beneficial role of catheter ablation only in the subgroup of patients with LVEF $\geq 25 \%$ ( $\mathrm{HR} 0.48 ; 95 \% \mathrm{Cl} 0.31-0.74$, but not in the subgroup of patients with LVEF <25\%) and in NYHA class II (HR 0.42; 95\% Cl 0.25-0.72, but not significant in the NYHA class III subgroup). Moreover, it has been postulated that the negative outcome of the AMICA trial was mostly driven by the unexpectedly high LVEF increase in the control arm, whereas the increase in LVEF in the catheter ablation arm was comparable to other studies ${ }^{84}$ Considering this finding, the prevalence of CRT-D and the high proportion of patients on a rhythm control strategy in the optimal medical therapy arm of the AMICA trial, it would be extremely interesting to assess whether the increase in LVEF in the optimal medical therapy arm was not driven by the resumption of adequate Bivp\% in patients having undergone CRT-D. However, the data on BiVp in the CRT-D subgroups of the AMICA trial are not available.

In this context, new evidence from randomized trials is urgently awaited. There are now at least two trials underway comparing rhythm and rate control in both the HF (RAFT-AF, NCT01420393) and the CRT populations (Pilot-CRAfT, NCT01850277). ${ }^{94-96}$ Their results should be published soon and may cast new light on the choice of the optimal AF treatment strategy in these patient subgroups.

Considering all of the aforementioned issues and inconsistencies, recently published ESC guidelines on the management of AF conclude that catheter ablation is recommended to reverse LV dysfunction in patients with AF when tachycardia-induced cardiomyopathy is highly probable (class I, level of evidence: B) and should be considered in selected patients with AF with HF with reduced LVEF to improve survival and reduce HF hospitalization (class Ila, level of evidence: B). ${ }^{68}$ Although not stated clearly, a choice for catheter ablation should be especially considered in patients with HF with NYHA class II symptoms and with an LVEF $>25 \%$ (unless the decrease in LVEF can be attributed to AF-induced cardiomyopathy), without other risk factors of a poorer catheter ablation outcome.

Importantly, the decision for rhythm control should be implemented promptly. Although they are based on the general AF population (not only patients with HF or having undergone CRT), the results of the recent EASTAFNET trial have shown that early introduction of rhythm control therapy within a year of AF diagnosis is associated with a reduction in the primary composite endpoint of cardiovascular death, stroke, hospitalizations associated with HF exacerbation and acute coronary syndrome. ${ }^{97}$

\section{Catheter ablation of atrial fibrillation in patients with heart failure: Gaps in evidence}

Current guidelines say nothing about the optimal choice or extent of the catheter ablation method. In all of the three aforementioned trials (CASTLE-AF, AMICA and CABANA), the only acceptable catheter ablation method was radiofrequency electrical current ablation, with PVI as an elementary component. However, in about half of the patients, additional 
sites (such as additional lines, ablation of complex fragmented atrial electrograms or ablation of AF triggers other than pulmonary veins) were ablated. Until now, there have been no convincing data that these additional ablation sites add to the efficacy of catheter ablation in the general AF population. However, their role in patients with HF undergoing catheter ablation has not been thoroughly examined yet.

Moreover, there are scarce data assessing cryoballoon PVI in the HF subpopulation. Consistent with the results of the FIRE AND ICE trial (ClinicalTrials.gov identifier: NCT01490814), which showed no significant difference between the radiofrequency ablation and cryoballoon PVI in the general, paroxysmal, AF population, the recent trials suggest similar feasibility and efficacy (in terms of LVEF increase) of the two catheter ablation methods in the HF subgroup. ${ }^{98-101}$ However, there is lack of evidence confirming the efficacy of cryoballoon ablation in terms of clinical endpoints, such as HF hospitalization risk or mortality, and new evidence is awaited.

Another unsolved issue is whether PVI and AVNA procedures should be performed alternatively or together. ${ }^{102}$ The questions are:

- to what extent will catheter ablation of AF in the CRT recipient be efficacious enough to provide adequate BiVp?; and

- is there any benefit in performing AF ablation in a patient undergoing CRT who has already undergone the AVNA procedure?

\section{Summary}

The incidence of AF in CRT recipients is very common, reaching 25-40\% of patients undergoing CRT. Although the management of AF in this group is no different to general population in terms of AF diagnosis principles, or the implementation and the choice of anticoagulants, close relations between AF and CRT efficacy determines the specificity of AF management in this subgroup of patients. The diagnosis of AF in patients treated with CRT negatively affects their prognosis. Moreover, $\mathrm{AF}$ is also a marker of poorer response to CRT. The surrogate of effective resynchronization in patients with $A F$ is the percentage of bi-ventricular effectively paced beats, which may be overestimated by a CRT device and should be as close to $100 \%$ as possible (preferably 298\%). In patients with AF and a BiVp\% <98\%, AVNA should be considered. Based on current scientific evidence, AVNA is the only treatment modality that positively affects the prognosis of a wide group of patients undergoing CRT with $A F$, and makes CRT efficacy comparable to the subgroup of patients with SR.

Apart from AVNA as a rate control strategy of AF treatment, there is a growing body of evidence supporting a rhythm control strategy with catheter ablation of AF as its foremost element also may be efficacious in this group of patients in terms of a better prognosis and lower mortality. It seems that among patients with HF who may profit the most from catheter ablation are those with a lower NYHA functional class, a higher EF and paroxysmal rather than long-standing persistent AF. However, the data showing a better prognosis and mortality reduction of patients undergoing catheter ablation come from the wide group of patients with HF. Unfortunately, the data comparing rhythm control with rate control strategies of AF treatment specific to the CRT subgroup are not available, and new evidence on this topic is urgently needed. $\square$
1. Khan NK, Goode KM, Cleland JG, et al. Prevalence of ECG abnormalities in an international survey of patients with suspected or confirmed heart failure at death or discharge. Eur J Heart Fail. 2007;9:491-501.

2. Khand AU, Rankin AC, Kaye GC, Cleland JG. Systematic review of the management of atrial fibrillation in patients with heart failure. Eur Heart J. 2000;21:614-32.

3. Maisel WH, Stevenson LW. Atrial fibrillation in heart failure: Epidemiology, pathophysiology, and rationale for therapy. Am J Cardiol. 2003;91:2D-8D.

4. Brignole $\mathrm{M}$, Auricchio $\mathrm{A}$, Baron-Esquivias $\mathrm{G}$, et al. 2013 ESC guidelines on cardiac pacing and cardiac resynchronization therapy: The task force on cardiac pacing and resynchronization therapy of the European Society of Cardiology (ESC). Developed in collaboration with the European Heart Rhythm Association (EHRA). Europace 2013;15:1070-118

5. Hayes DL, Boehmer JP, Day JD, et al. Cardiac resynchronization therapy and the relationship of percent biventricular pacing to symptoms and survival. Heart Rhythm. 2011;8:1469-75.

6. Ousdigian KT, Borek PP, Koehler JL, et al. The epidemic of inadequate biventricular pacing in patients with persistent or permanent atrial fibrillation and its association with mortality. Circ Arrhythm Electrophysiol. 2014;7:370-6.

7. Dickstein K, Normand C, Auricchio A, et al. CRT Survey II: A European Society of Cardiology survey of cardiac II: A European Society of Cardiology survey of cardiac
resynchronisation therapy in 11,088 patients - who is doing what to whom and how? Eur J Heart Fail. 2018;20:1039-51.

8. Łasocha D, Sterliński M, Tajstra M, et al. Do we differ in terms of indications and demographics in cardiac resynchronisation recipients in Poland? Insights from the European CRT Survey II Registry. Kardiol Pol. 2019;77:40-6.

9. Ponikowski P, Voors AA, Anker SD, et al. 2016 ESC Guidelines or the diagnosis and treatment of acute and chronic heart failure: The Task Force for the diagnosis and treatment of acute and chronic heart failure of the European Society of Cardiology (ESC) developed with the special contribution of the Heart Failure Association (HFA) of the ESC. Eur Heart J. 2016;37:2129-200

10. Yancy CW, Jessup M, Bozkurt B, et al. 2013 ACCF/AHA guideline for the management of heart failure: $A$ report of the American College of Cardiology Foundation/American Heart Association Task Force on practice guidelines. Circulation. 2013;128:e240-327.

11. Kusumoto FM, Schoenfeld MH, Barrett C, et al. 2018 ACC/AHA HRS guideline on the evaluation and management of patients with bradycardia and cardiac conduction delay: A report of the American College of Cardiology/American Heart Association Task Force on Clinical Practice Guidelines and the Heart Rhythm Society. J Am Coll Cardiol. 2019;74:e51-156.

12. Moss AJ, Hall WJ, Cannom DS, et al. Cardiac-resynchronization therapy for the prevention of heart-failure events. N Eng/ I Med. 2009:361:1329-38

13. Linde C, Abraham WT, Gold MR, et al. Randomized trial of cardiac resynchronization in mildly symptomatic heart failure patients and in asymptomatic patients with left ventricular dysfunction and previous heart failure symptoms. J Am Coll Cardiol. 2008;52:1834-43.

14. Daubert C, Gold MR, Abraham WT, et al. Prevention of disease progression by cardiac resynchronization therapy in patients with asymptomatic or mildly symptomatic left ventricular dysfunction: Insights from the European cohort of the REVERSE (Resynchronization Reverses Remodeling in Systolic Left Ventricular Dysfunction) trial. J Am Coll Cardiol. 2009;54:1837-46.

15. Dickstein K Vardas PE Auricchio A et al. 2010 Focused update of ESC guidelines on device therapy in heart failure: An update of the 2008 ESC guidelines for the diagnosis and treatment of acute and chronic heart failure and the 2007 ESC guidelines for cardiac and resynchronization therapy. Developed with the special contribution of the Heart Failure Association and the European Heart Rhythm Association. Eur Heart 2010;31:2677-87.

16. ClinicalTrialss.gov. MADIT-CRT: Multicenter Automatic Defibrillator Implantation With Cardiac Resynchronization Therapy (MADITCRT) . ClinicalTrials.gov Identifier: NCT00180271. Available at: www.clinicaltrials.gov/ct2/show/NCT00180271 (accessed 18 June 2021).

17. ClinicalTrials.gov. REsynchronization reVErses Remodeling in Systolic Left vEntricular Dysfunction (REVERSE). ClinicalTrials gov Identifier. NCTO0271154. Available at: https://clinicaltrials. gov/ct2/show/NCT00271154 (accessed 18 June 2021).

18. Leclercq $C$, Walker $S$, Linde $C$, et al. Comparative effects of permanent biventricular and right-univentricular pacing in heart failure patients with chronic atrial fibrillation. Eur Heart 2002;23:1780-7.

19. Ebert $\mathrm{M}$, Jander $\mathrm{N}$, Minners J, et al. Long-term impact of right ventricular pacing on left ventricular systolic function in pacemaker recipients with preserved ejection fraction: Results from a large single-center registry. J Am Heart Assoc. 2016;5:e003485.

20. Veasey RA, Arya A, Silberbauer J, et al. The relationship between right ventricular pacing and atrial fibrillation burden and disease progression in patients with paroxysmal atrial fibrillation: The long-MinVPACE study. Europace. 2011:13:815-20.

21. Ciszewski JB, Chwyczko T, Kowalik I, et al. Clinical echocardiographic, and pacing parameters affecting atrial fibrillation burden in patients with tachycardia-bradycardia syndrome. Kardiol Pol. 2018;76:338-6.

22. Silberbauer J, Veasey RA, Freemantle $\mathrm{N}$, et al. The relationship between high-frequency right ventricular pacing and paroxysmal atrial fibrillation burden. Europace. 2009;11:1456-61.

23. Sweeney MO, Hellkamp AS, Ellenbogen KA, et al. Adverse effect of ventricular pacing on heart failure and atrial fibrillation among patients with normal baseline QRS duration in a clinical trial of pacemaker therapy for sinus node dysfunction. Circulation. 2003;107:2932-7.
24. Sweeney MO, Bank AJ, Nsah E, et al. Minimizing ventricular pacing to reduce atrial fibrillation in sinus-node disease. N Engl J Med. 2007;357:1000-8.

25. Smit MD, Van Dessel PF, Nieuwland W, et al. Right ventricular pacing and the risk of heart failure in implantable cardioverter-defibrillator patients. Heart Rhythm. 2006;3:1397-403.

26. Tang AS, Wells GA, Talajic M, et al. Cardiac-resynchronization therapy for mild-to-moderate heart failure. N Eng/ J Med. 2010;363:2385-95

27. Healey JS, Hohnloser SH, Exner DV, et al. Cardiac resynchronization therapy in patients with permanent atrial fibrillation: Results from the Resynchronization for Ambulatory Heart Failure Trial (RAFT). Circ Heart Fail. 2012;5:566-70.

28. Bristow MR, Saxon LA, Boehmer J, et al. Cardiac-resynchronization therapy with or without an implantable defibrillator in advanced chronic heart failure. N Eng/ J Med. 2004;350:2140-50

29. Kalscheur MM, Saxon LA, Lee BK, et al. Outcomes of cardiac resynchronization therapy in patients with intermittent atrial fibrillation or atrial flutter in the COMPANION trial. Heart Rhythm. 2017;14:858-65.

30. Upadhyay GA, Choudhry NK, Auricchio A, et al. Cardiac resynchronization in patients with atrial fibrillation: A meta-analysis of prospective cohort studies. J Am Coll Cardiol. 2008:52:1239-46.

31. Wilton $S B$, Leung $A A$, Ghali WA, et al. Outcomes of cardiac resynchronization therapy in patients with versus those without atrial fibrillation: A systematic review and meta-analysis. Heart Rhythm. 2011;8:1088-94.

32. Khazanie P, Greiner MA, Al-Khatib SM, et al. Comparative effectiveness of cardiac resynchronization therapy among patients with heart failure and atrial fibrillation: Findings from the National Cardiovascular Data Registry's Implantable Cardioverter-Defibrillator Registry. Circ Heart Fail. 2016;9:10.1161/CIRCHEARTFAILURE.115.002324 e002324.

33. van Boven $N$, Theuns $D$, Bogaard $K$, et al. Atrial fibrillation in cardiac resynchronization therapy with a defibrillator: A risk factor for mortality, appropriate and inappropriate shocks. J fardiovasc Electrophysiol. 2013:24:1116-22.

34. Tolosana JM, Hernandez Madrid A, Brugada J, et al. Comparison of benefits and mortality in cardiac resynchronization therapy in patients with atrial fibrillation versus patients in sinus rhythm (results of the Spanish Atrial Fibrillation and Resynchronization [SPARE] study). Am J Cardiol. 2008;102:444-9.

35. Tolosana JM, Arnau AM, Madrid AH, et al. Cardiac resynchronization therapy in patients with permanent atrial fibrillation. Is it mandatory to ablate the atrioventricular junction to obtain a good response? Eur J Heart Fail. 2012;14:635-41.

36. Khadjooi K, Foley PW, Chalil S, et al. Long-term effects of cardiac resynchronisation therapy in patients with atrial fibrillation. Heart. 2008;94:879-83.

37. Köbe J, Wasmer K, Andresen D, et al. Impact of atrial fibrillation on early complications and one year-survival after cardioverter 
defibrillator implantation: Results from the German DEVICE registry. Int J Cardiol. 2013:168:4184-90.

38. Mustafa U, Atkins I, Mina G, et al Outcomes of cardiac resynchronisation therapy in patients with heart failure with atrial fibrillation: A systematic review and meta-analysis of observational studies. Open Heart. 2019;6:e000937.

39. Boriani G, Gasparini M, Landolina M, et al. Incidence and clinical relevance of uncontrolled ventricular rate during atrial fibrillation in heart failure patients treated with cardiac resynchronization therapy. Eur J Heart Fail. 2011;13:868-76.

40. Koplan BA, Kaplan AJ, Weiner S, et al. Heart failure decompensation and all-cause mortality in relation to percent biventricular pacing in patients with heart failure: Is a goal of $100 \%$ biventricular pacing necessary? J Am Coll Cardiol. 2009;53:355-60.

41. Mazurek M, Jędrzejczyk-Patej E, Lenarczyk R, et al. Do we need to monitor the percentage of biventricular pacing day by day? Int I Cardiol. 2016:221:81-9.

42. Ruwald AC, Kutyifa V Ruwald MH, et al. The association between biventricular pacing and cardiac resynchronization therapy-defibrillator efficacy when compared with implantable cardioverter defibrillator on outcomes and reverse remodelling Eur Heart J. 2015;36:440-8.

43. Kamath GS, Cotiga D, Koneru JN, et al. The utility of 12-lead Holter monitoring in patients with permanent atrial fibrillation for the identification of nonresponders after cardiac resynchronization therapy. J Am Coll Cardiol. 2009;53:1050-5.

44. Hernández-Madrid A, Lu X, Tsintzos SI, et al. Heart failure hospitalization reduction and cost savings achieved by improved delivery of effective biventricular pacing: economic implications of the OLE study under the US setting. Clinicoecon Outcomes Res. 2019:11:385-93.

45. Gasparini M, Regoli F, Galimberti P, et al. Cardiac resynchronization therapy in heart failure patients with atrial fibrillation. Europace. 2009:11(Suppl. 5):v82-6.

46. Ciaramitaro $G$, Sgarito $G$, Solimene $F$, et al. Role of rate contro and regularization through pacing in patients with chronic atria fibrillation and preserved ventricular function: The VRR study. Pacing Clin Electrophysiol. 2006;29:866-74.

47. Plummer CJ, Frank CM, Bári Z, et al. A novel algorithm increase the delivery of effective cardiac resynchronization therapy during atrial fibrillation: The CRTee randomized crossover trial. Heart Rhythm. 2018;15:369-75.

48. Jafferani A, Leal MA. Advances in cardiac resynchronization therapy. J Innov Card Rhythm Manag. 2019;10:3681-93.

49. Gasparini M, Auricchio A, Regoli F, et al. Four-year efficacy of cardiac resynchronization therapy on exercise tolerance and disease progression: The importance of performing atrioventricular junction ablation in patients with atrial fibrillation. J Am Coll Cardiol. 2006:48:734-43.

50. Gasparini M, Auricchio A, Metra M, et al. Long-term survival in patients undergoing cardiac resynchronization therapy: The importance of performing atrio-ventricular junction ablation in patients with permanent atrial fibrillation. Eur Heart 2008;29:1644-52.

51. Gasparini M, Leclercq C, Lunati M, et al. Cardiac resynchronization therapy in patients with atrial fibrillation: The CERTIFY study (Cardiac Resynchronization Therapy in Atrial Fibrillation Patients Multinational Registry). JACC Heart Fail. 2013:1:500-7.

52. Ferreira AM, Adragão P, Cavaco DM, et al. Benefit of cardiac resynchronization therapy in atrial fibrillation patients vs. patients in sinus rhythm: The role of atrioventricular junction ablation. Europace. 2008:10:809-15.

53. Dong K, Shen $\mathrm{W}-\mathrm{K}$, Powell $\mathrm{BD}$, et al. Atrioventricular nodal ablation predicts survival benefit in patients with atrial fibrillation receiving cardiac resynchronisation therapy. Heart Rhythm. 2010;7:1240-5.

54. Mittal S, Musat DL, Hoskins MH, et al. Clinical outcomes after ablation of the AV junction in patients with atrial fibrillation: Impact of cardiac resynchronization therapy. J Am Heart Assoc. 2017;6:e007270

55. Jędrzejczyk-Patej E, Lenarczyk R, Pruszkowska P, et al. Long-term outcomes of cardiac resynchronization therapy are worse in patients who require atrioventricular junction ablation for atrial fibrillation than in those with sinus rhythm. Cardiol J. 2014;21:309-15.

56. Xue Y, Wang J, Thapa S, et al. Comparison of survival for cardiac resynchronization therapy in atrial fibrillation patients with or without atrio-ventricular junction ablation and patients in sinus rhythm: A systematic review and network meta-analysis. Heart Fail Rev. 2019;24:335-42.

57. Kindermann M, Hennen B, Jung J, et al. Biventricular versus conventional right ventricular stimulation for patients with standard pacing indication and left ventricular dysfunction: The Homburg Biventricular Pacing Evaluation (HOBIPACE). J Am Coll Cardiol. 2006;47:1927-37.

58. Curtis AB, Worley SJ, Adamson PB, et al. Biventricular pacing for atrioventricular block and systolic dysfunction. N Eng/ J Med. 2013;368:1585-93

59. ClinicalTrials.gov. Biventricular Versus Right Ventricular Pacing in Heart Failure Patients With Atrioventricular Block (BLOCK HF). ClinicalTrials.gov Identifier: NCT00267098. Available at: https://clinicaltrials.gov/ct2/show/NCT00267098 (accessed 18 June 2021).

60. Curtis AB, Worley SJ, Chung ES, et al. Improvement in clinica outcomes with biventricular versus right ventricular pacing: The BLOCK HF study. J Am Coll Cardiol. 2016;67:2148-57.

61. St John Sutton M, Plappert T, Adamson PB, et al. Left ventricula reverse remodeling with biventricular versus right ventricular pacing in patients with atrioventricular block and heart failure in the BLOCK HF trial. Circ Heart Fail. 2015;8:510-18

62. Leon AR, Greenberg JM, Kanuru N, et al. Cardiac resynchronization in patients with congestive heart failure and chronic atrial fibrillation: Effect of upgrading to biventricular pacing after chronic right ventricular pacing. J Am Coll Cardio. 2002;39:1258-63

63. Slotwiner DJ, Raitt MH, Del-Carpio Munoz F, et al. Impact of physiologic pacing versus right ventricular pacing among patients with left ventricular ejection fraction greater than $35 \%$ A systematic review for the 2018 ACC/AHA/HRS guideline on the evaluation and management of patients with bradycardia and cardiac conduction delay: a report of the American college of Cardiology/American Heart Association Task Force on Clinical Practice Guidelines and the Heart Rhythm Society. Circulation. 2019;140:e483-503.

64. Kiehl EL, Makki T, Kumar R, et al. Incidence and predictors of right ventricular pacing-induced cardiomyopathy in patients with complete atrioventricular block and preserved left ventricular systolic function. Heart Rhythm 2016;13:2272-8

65. Stockburger M, Gómez-Doblas JJ, Lamas G, et al. Preventing ventricular dysfunction in pacemaker patients without advanced heart failure: Results from a multicentre international randomized trial (PREVENT-HF). Eur J Heart Fail. 2011:13:633-41.

66. Packer DL, Mark DB, Robb RA, et al. Effect of catheter ablation vs antiarrhythmic drug therapy on mortality, stroke, bleeding, and cardiac arrest among patients with atrial fibrillation: The CABANA randomized clinical trial. JAMA. 2019;321:1261-74.

67. Sethi NJ, Feinberg J, Nielsen EE, et al. The effects of rhythm control strategies versus rate control strategies for atria fibrillation and atrial flutter: A systematic review with meta-analysis and trial sequential analysis. PLOS One. 2017; $12: \mathrm{e} 0186856$

68. Hindricks G, Potpara T, Dagres N, et al. 2020 ESC guidelines for the diagnosis and management of atrial fibrillation developed in collaboration with the European Association of Cardio-Thoracic Surgery (EACTS). Eur Heart J. 2020:ehaa612.

69. Pang H, Ronderos R, Pérez-Riera AR, et al. Reverse atrial electrical remodeling: a systematic review. Cardiol $\mathrm{f}$ 2011:18:625-31.

70. Sutton MS, Keane MG. Reverse remodelling in heart failure with cardiac resynchronisation therapy. Heart. 2007;93:167-71

71. Gasparini M, Steinberg JS, Arshad A, et al. Resumption of sinus rhythm in patients with heart failure and permanent atrial fibrillation undergoing cardiac resynchronization therapy: A
Iongitudinal observational study. Eur Heart J. 2010;31:976-83.

72. Delnoy PP, Ottervanger JP, Luttikhuis HO, et al. Comparison of usefulness of cardiac resynchronization therapy in patients with atrial fibrillation and heart failure versus patients with sinus rhythm and heart failure. Am I Cardiol. 2007:99:1252-7.

73. ClinicalTrials.gov. Care-HF CArdiac Resynchronization in Heart Failure. ClinicalTrials.gov Identifier: NCT00170300. Available at: https://clinicaltrials.gov/ct2/show/NCT00170300 (accessed 22 July 2021).

74. ClinicalTrials gov Resynchronization/Defibrillation for Ambulatory Heart Failure Trial (RAFT). ClinicalTrials.gov Identifier: NCT00251251. Available at: wuw clinicaltrials gov/ct2/show/ NCT00251251 (accessed 22 July 2021).

75. Borleffs CJ, Ypenburg C, van Bommel RJ, et al. Clinical importance of new-onset atrial fibrillation after cardiac resynchronization therapy. Heart Rhythm. 2009;6:305-10.

6. Hoppe UC, Casares JM, Eiskjaer H, et al. Effect of cardiac resynchronization on the incidence of atrial fibrillation in patients with severe heart failure. Circulation. 2006;114:18-25.

77. Adelstein EC, Saba S. Burden of atrial fibrillation after cardiac resynchronization therapy. Am I Cardiol. 2007;100:268-72.

78. Birnie D, Hudnall H, Lemke B, et al. Continuous optimization of cardiac resynchronization therapy reduces atrial fibrillation in heart failure patients: Results of the Adaptive Cardiac Resynchronization Therapy trial. Heart Rhythm. 2017:14:1820-5.

79. Hsu JC, Birnie D, Stadler RW et al. Adaptive cardiac resynchronization therapy is associated with decreased risk of incident atrial fibrillation compared to standard biventricula pacing: A real-world analysis of 37,450 patients followe
remote monitoring. Heart Rhythm. 2019:16:983-9.

80. Ecker V, Knoery C, Rushworth G, et al. A review of factors associated with maintenance of sinus rhythm after electiv electrical cardioversion for atrial fibrillation. Clin Cardiol. 2018;41:862-70.
81. ClinicalTrials.gov. Atrioventricular Junction Ablation and Biventricular Pacing for Atrial Fibrillation and Heart Failure (APAF-CRT). ClinicalTrials.gov Identifier: NCT02137187. Available at: https://clinicaltrials.gov/ct2/show/NCT02137187 (accessed 18 June 2021).

82. Marrouche NF, Brachmann J, Andresen D, et al. Catheter ablation for atrial fibrillation with heart failure. N Eng/ J Med. 2018;378:417-27.

83. Packer DL, Monahan KH, Al-Khalidi HR, et al. Ablation of atrial fibrillation in heart failure patients: Additional outcomes of the CABANA Trial. Heart Rhythm. 2019.16(Suppl. 1):Abstract S-AB-14-06.

84. Kuck KH, Merkely B, Zahn R, et al. Catheter ablation versus best medical therapy in patients with persistent atrial fibrillation and congestive heart failure: The randomized AMICA trial. Circ Arrhythm Electrophysiol. 2019;12:e007731.

85. Wyse DG, Waldo AL, DiMarco JP, et al. A comparison of rate control and rhythm control in patients with atrial fibrillation. N Engl J Med. 2002;347:1825-33.

86. Roy D, Talajic M, Nattel S, et al. Rhythm control versus rate control for atrial fibrillation and heart failure. N Eng/ I Med. 2008:358:2667-77.

87. Zhao Y, Krupadev V, Dagher L, et al. Pharmacological rhythm versus rate control in patients with atrial fibrillation and heart failure: The CASTLE-AF trial. J Interv Card Electrophysiol. 2020. DOI:10.1007/s10840-020-00856-1. [Online ahead of print].

88. Jones DG, Haldar SK, Hussain W, et al. A randomized tria to assess catheter ablation versus rate control in the management of persistent atrial fibrillation in heart failure J Am Coll Cardiol. 2013;61:1894-903.

89. Hunter RJ, Berriman TJ, Diab I, et al. A randomized controlled trial of catheter ablation versus medical treatment of atria fibrillation in heart failure (the CAMTAF trial). Circ Arrhythm Electrophysiol. 2014;7:31-8

90. Khan $\mathrm{MN}$, Jaïs $\mathrm{P}$, Cummings J, et al. Pulmonary-vein isolation for atrial fibrillation in patients with heart failure. N Eng/ I Med 2008;359:1778-85

91. Di Biase L, Mohanty P, Mohanty S, et al. Ablation versus amiodarone for treatment of persistent atrial fibrillation in patients with congestive heart failure and an implanted device: Results from the AATAC multicenter randomized trial. Circulation. 2016;133:1637-44.

92. ClinicalTrials.gov. Catheter Ablation vs Anti-arrhythmic Drug Therapy for Atrial Fibrillation Trial (CABANA). ClinicalTrials.gov Identifier: NCT00911508. Available at: https://clinicaltrials.gov/ ct2/show/NCT00911508 (accessed 22 July 2021).

93. ClinicalTrials.gov. Atrial Fibrillation Management in Congestive Heart Failure With Ablation (AMICA). ClinicalTrials.gov Identifier: NCT00652522. Available at: https://clinicaltrials.gov/ct2/show/ NCT00652522 (accessed 22 July 2021).

94. Ciszewski J, Maciag A, Kowalik I, et al. Comparison of the rhythm control treatment strategy versus the rate control strategy in patients with permanent or long-standing persistent atrial fibrillation and heart failure treated with cardiac resynchronization therapy - a pilot study of Cardiac Resynchronization in Atrial Fibrillation Trial (Pilot-CRAfT): Study protocol for a randomized controlled trial. Trials. 2014;15:386.

95. ClinicalTrials.gov. Rhythm control - catheter ablation with or without anti-arrhythmic drug control of maintaining sinus rhythm versus rate control with medical therapy and/or atrioventricular junction ablation and pacemaker treatment for atria fibrillation (RAFT-AF). ClinicalTrials.gov Identifier: NCT01420393. Available at: https://clinicaltrials.gov/ct2/show/NCT01420393 (accessed 21 May 2021).

96. ClinicalTrials. gov. Cardiac resynchronization in atrial fibrillation trial - a pilot study (Pilot-CRAfT). ClinicalTrials.gov Identifier: NCT01850277. Available at: https://clinicaltrials.gov/ct2/show/ NCT01850277 (accessed 21 May 2021).

97. Kirchhof P, Camm AJ, Goette A, et al. Early rhythm-control therapy in patients with atrial fibrillation. N Eng/ J Med. 2020;383:1305-6.

98. ClinicalTrials.gov. FIRE AND ICE: Comparative Study of Two Ablation Procedures in Patients With Atrial Fibrillation. ClinicalTrials.gov Identifier: NCT01490814. Available at: www.clinicaltrials.gov/ct2/show/NCT01490814 (accessed 22 July 2021).

99. Kuck KH, Brugada J, Fürnkranz A, et al. Cryoballoon or radiofrequency ablation for paroxysmal atrial fibrillation. N Eng/ J Med. 2016;374:2235-45.

100. Pruszkowska P, Lenarczyk R, Gumprecht J, et al. Cryoballoon ablation of atrial fibrillation in patients with advanced systolic heart failure and cardiac implantable electronic devices. Kardiol Pol. 2018:76:1081-8.

101. Pott A, Jäck S, Schweizer $C$, et al. Atrial fibrillation ablation in heart failure patients: improved systolic function after cryoballoon pulmonary vein isolation. ESC Heart Fail. 2020;7:2258-67.

102. Ciszewski J, Sterlinski M. Rhythm control and AV node ablation in CRT patients with atrial fibrillation: should they be performed together or alternatively? Pacing Clin Electrophysio 2015;38:906-7. 